Akira Imamura • Sumihisa Honda • Yoshibumi Nakane Yuji Okazaki

\title{
Anticipation in Japanese families with schizophrenia
}

\begin{abstract}
The identification of anticipation in schizophrenia is a recent focus in the genetic epidemiology of schizophrenia, although it involves some controversial methodological issues. We explored the evidence of anticipation among 44 Japanese two-generation pairs with schizophrenia found by reviewing nine years of admission records (1986-1994) at the Department of Neuropsychiatry, Nagasaki University Hospital and Michino-o Hospital, Nagasaki. The 44 pairs consisted of 27 two-generation pairs of first-degree relatives group (FDRG) and 17 pairs of second-degree relatives group (SDRG). On pairwise comparison and a life table analysis, the age at onset (AO) was significantly earlier in the lower generation (G2) than in the upper generation (G1) in all of the pairs and in the two subgroups, FDRG and SDRG. Earlier AO was shown in G2 even after minimizing some statistical biases for the study of anticipation in schizophrenia. A significant earlier mean AO was found in $\mathrm{G} 2$ even when a cohort effect was controlled for. There was no marked difference in AO between paternal and maternal transmission. These results provide further evidence for epidemiological anticipation, suggesting biological anticipation such as the involvement of trinucleotide repeats expansion in G2. The limitations of the study are also discussed.
\end{abstract}

Key words Anticipation - Schizophrenia $\cdot$ Ascertainment bias - Cohort effect - Trinucleotide repeats expansion . Affected two-generation pair method $\cdot$ Genomic imprinting

\footnotetext{
A. Imamura $(\bowtie) \cdot$ Y. Nakane $\cdot$ Y. Okazaki

Department of Neuropsychiatry, Nagasaki University School of

Medicine, Sakamoto 1-7-1, Nagasaki 852-8501, Japan

Tel. +81-95-849-7293; Fax +81-95-849-7296

e-mail: f1042@cc.nagasaki-u.ac.jp

S. Honda

Department of Radiation Epidemiology, Atomic Bomb Disease Institute, Nagasaki University School of Medicine, Nagasaki, Japan
}

This paper is based in part on a presentation made at the Annual Meeting of the Japan Society of Human Genetics, at Kumamoto, October 1995, and at the World Congress of Psychiatric Genetics, San Francisco, October 1996.

\section{Introduction}

Anticipation is a clinical genetic concept that has been epidemiologically defined as a decrease in age at onset (AO) of the disease and an increase in severity in succeeding generations. Recently, the correlation of epidemiological anticipation with biological anticipation, in terms of trinucleotide repeats expansion (TRE), has been demonstrated in several neuromuscular and neurological diseases (Harper et al. 1992, Ashley and Warren 1995). This validates the genetic concept of anticipation after a long period of neglect. The dismissal of the concept in genetics was provoked after rigorous methodological criticism by Penrose (1948) in response to inflated reports of anticipation in some neuropsychiatric disorders. He regarded them as artifacts that were caused by ascertainment biases.

However, since the late 1980s, new evidence of epidemiological anticipation in several neuromuscular and neurological diseases has been reported, even after ascertainment biases were excluded (Ridley et al. 1988; Höweler et al. 1989), and these lines of evidence were soon followed by evidence of biological anticipation that showed a correlation between decrease in $\mathrm{AO}$ and increase in severity with the presence of TRE. These findings provide not only a new understanding of the molecular-genetic basis of anticipation in those diseases but also suggest a reconsideration of the involvement of anticipation in psychiatric disorders. Consequently, anticipation in schizophrenia has been explored recently in both epidemiological and moleculargenetic studies (Bassett and Honer 1994).

Several researchers who have minimized ascertainment biases and overcome methodological problems such as cohort effect and bilineality, have demonstrated a decrease in $\mathrm{AO}$ and an increase in severity in lower generations of schizophrenia patients, (Bassett and Honer 1994; Stöber et al. 1995; Thibaut et al. 1995; Gorwood et al. 1996; Johnson et al. 1997). On the other hand, doubts have been cast on data which showed anticipation in schizophrenia (Asherson et al. 1994; Yaw et al. 1996) and other familial disorders (Heiman et al. 1996; Fraser 1997). 
In molecular-genetics research on anticipation, O'Donovan et al. (1995) have used the repeat expansion detection (RED) method to show longer CAG repeats in the DNA of schizophrenia patients than in the DNA of normal controls. Their report, however, has been followed by reports of conflicting findings (Dann et al. 1996; Laurent et al. 1996; Petronis et al. 1996; Vincent et al. 1996). Nakamoto et al. (1997) have identified long CAG/CTG repeats in the normal population, and have suggested that such repeats would contribute to most of the false-negative results and should be excluded in the RED experiments. Recently, a genomic discordance between monozygotic twins discordant and concordant for schizophrenia has been reported, and these differences could be due to the presence of TRE (Broude et al. 1997).

Thus, the evidence for epidemiological and biological anticipation in schizophrenia is still inconclusive and requires further investigation. In the present study, we compared AO in 44 two-generation pairs with schizophrenia to explore evidence for epidemiological anticipation in schizophrenia. To cope with some reported methodological problems, such as bilineality, ascertainment biases, and a cohort effect (Table 1), we systematically applied several measures, including a prediction of morbidity risk among unaffected siblings of patients with schizophrenia. We also highlight the possibility that a skewed female-to-male ratio may mimic anticipation.

\section{Subjects and methods}

The following method was approved by the Ethics Committee of Nagasaki University School of Medicine. Probands were defined as DSM-IV (Diagnostic and Statistical Manual of Mental Disorders, 4th edition) schizophrenia patients with at least one first or second degree relative, in the upper generation (G1) or lower generation (G2) (father, mother, child, uncle, aunt, nephew, or niece) who suffered from psychosis, including schizophrenia, schizophreniform disorder, schizoaffective disorder, delusional disorder, brief psychotic disorder, or psychotic disorder not otherwise specified. Excluded were mood disorders, psychotic disorder due to general medical condition, substance-induced psychotic disorder, and suicide without psychotic symptoms. Probands were drawn from 863 Japanese schizophrenia patients consecutively admitted to the Department of Neuropsychiatry, Nagasaki University Hospital and Michino-o Hospital, Nagasaki, between 1986 and 1994. We reviewed their hospital admission records and found 86 probands. All hospital records reviewed included sufficient information for this study because, in both the hospitals, at least two different doctors or a doctor and a clinical psychologist independently took histories of past, family, and present illness from both maternal and paternal informants. The group whose probands had at least one member who suffered from psychosis among their first degree relatives (FDRG, first degree relatives group) included 50 probands. The group whose probands had at least one
Table 1 Problems in the epidemiological study of anticipation in schizophrenia

1. Bilineality, assortative mating (Bassett 1994; Stöber 1995)

2. Random sampling, samples with age over the risk period (this study)

3. Ascertainment bias:

(i) Proband effect (Penrose 1948)

(ii) Simultaneous onset effect (Penrose 1948)

(iii) Risk for siblings of developing schizophrenia (this study)

(iv) Discordant ATA ${ }^{\mathrm{a}}$ between $\mathrm{G} 1^{\mathrm{b}}$ and $\mathrm{G} 2^{\mathrm{c}}$ (Gorwood 1996)

4. Truncation bias (Fraser 1997) or reduced fertility effect (Penrose 1948) $)^{\mathrm{d}}$

5. Cohort effect, period effect (Bassett 1994)

6. Skewed female-to-male ratio (this study)

${ }^{\text {a }}$ Age at time of ascertainment.

${ }^{\mathrm{b}}$ Upper generation.

${ }^{\mathrm{c}}$ Lower generation.

${ }^{\mathrm{d}}$ Although Penrose (1948) demonstrated that this was an

ascertainment bias, Fraser (1997) called it a "truncation bias" and distinguished it from ascertainment biases.

Table 2 Included and excluded probands

\begin{tabular}{lcccc}
\hline & \multicolumn{2}{c}{ Probands } & & \\
\cline { 2 - 3 } & Included & Excluded & $\begin{array}{l}\text { Test } \\
\text { statistics }\end{array}$ & $P$ value \\
\hline Sex & $n=21 \mathrm{M} ;$ & $n=23 \mathrm{M} ;$ & $\chi^{2}=0.813$ & 0.3672 \\
& $n=16 ; \mathrm{F}$ & $n=26 ; \mathrm{F}$ & & \\
Mean ATA (SD) & $40.8(13.5)$ & $41.9(14.7)$ & $U=875.0$ & 0.7834 \\
Mean AO (SD) & $22.6(7.2)$ & $25.6(10.4)$ & $U=775.0$ & 0.2504 \\
\hline
\end{tabular}

M, Male; F, female; $U$, Mann-Whitney's $U$-test; ATA, age at time of ascertainment (years), AO, age at onset of the disease (years)

family member who suffered from psychosis among second degree relatives (SDRG, second degree relatives group) included 41 probands. five probands were included in both groups. We excluded 8 probands due to bilineal transmission, and 41 probands because we selected only probands whose affected relative(s) were interviewed by a psychiatrist and had medical records which included sufficient information. Therefore, all subjects were directly interviewed by at least one doctor. A total of 37 probands were included in the study (27 in FDRG, 11 in SDRG; 1 proband was included in both groups). In pedigrees with two or more members who suffered from psychosis in a generation, we extracted all possible pairs. For the final analysis, we had 27 pairs in FDRG (24 patients in G1 and 27 patients in G2) and 17 pairs in SDRG (15 patients in G1 and 12 patients in G2). All subjects are Japanese. Table 2 summarizes the information for included and excluded probands. The included and excluded groups showed no significant differences in AO, sex ratios, and age at the time of ascertainment (ATA). Our sample was not skewed by excluding some probands.

$\mathrm{AO}$ was defined as the age of the patient at the onset of psychotic symptoms that allowed for diagnosis. We know no valid way of rating schizophrenia severity, because no appropriate criterion has been developed to estimate the disease's lifetime severity. We considered that the AO in schizophrenia reflected the disease severity to some degree.

We compared AO in G1 and G2 in all of the pairs and in the two subgroups, FDRG and SDRG, using the Wilcoxon 
Table 3 Pairwise comparisons of AO (years) in G1 and G2

\begin{tabular}{|c|c|c|c|c|c|c|c|c|c|c|c|c|}
\hline \multirow[b]{2}{*}{ Subjects } & \multirow[b]{2}{*}{$\begin{array}{l}\text { Type } \\
\text { of pairs }\end{array}$} & \multirow[b]{2}{*}{$\begin{array}{l}\text { Number } \\
\text { of pairs }\end{array}$} & \multicolumn{4}{|l|}{ G1 } & \multicolumn{4}{|l|}{ G2 } & \multirow[b]{2}{*}{ Z } & \multirow[b]{2}{*}{$P$ value } \\
\hline & & & Proband & $\begin{array}{l}\text { MAO } \\
(\mathrm{SD})\end{array}$ & Range & $\begin{array}{l}\text { MATA } \\
(\mathrm{SD})\end{array}$ & Proband & $\begin{array}{l}\text { MAO } \\
(\mathrm{SD})\end{array}$ & Range & $\begin{array}{l}\text { MATA } \\
\text { (SD) }\end{array}$ & & \\
\hline \multirow[t]{3}{*}{ All pairs } & Total & 44 & 6 & $35.1(11.0)$ & $15-59$ & $66.5(12.0)$ & 36 & $20.3(5.9)$ & $12-34$ & $37.8(9.5)$ & -5.72 & $<0.0001$ \\
\hline & FDRG & 27 & 5 & $38.2(10.4)$ & $15-59$ & $68.6(10.0)$ & 22 & $21.0(5.8)$ & $14-34$ & $38.2(9.8)$ & -4.48 & $<0.0001$ \\
\hline & SDRG & 17 & 1 & $30.2(10.4)$ & $15-47$ & $63.2(14.4)$ & 14 & $19.0(6.0)$ & $12-34$ & $37.1(9.3)$ & -3.62 & 0.0003 \\
\hline \multirow{3}{*}{$\begin{array}{l}\text { Outliers } \\
\text { excluded } \\
\text { pairs }\end{array}$} & Total & 20 & 3 & $31.7(6.4)$ & $22-40$ & $66.0(12.7)$ & 20 & $22.1(4.1)$ & $17-33$ & $36.1(8.5)$ & -3.81 & 0.0001 \\
\hline & FDRG & 11 & 2 & $33.5(5.4)$ & $23-40$ & $68.3(8.7)$ & 11 & $22.9(4.6)$ & $17-33$ & $38.7(7.6)$ & -2.80 & 0.0050 \\
\hline & SDRG & 9 & 1 & $29.4(7.1)$ & $22-40$ & $63.1(16.5)$ & 9 & $21.0(3.2)$ & $18-28$ & $32.8(8.7)$ & -2.67 & 0.0076 \\
\hline \multirow{3}{*}{$\begin{array}{l}\text { Narrow } \\
\text { diagnosed } \\
\text { pairs }\end{array}$} & Total & 34 & 5 & $32.7(10.1)$ & $15-49$ & $67.4(12.4)$ & 27 & $20.5(6.3)$ & $12-34$ & $39.5(9.7)$ & -5.00 & $<0.0001$ \\
\hline & FDRG & 18 & 4 & $35.6(9.1)$ & $15-49$ & $70.5(9.5)$ & 14 & $21.8(6.2)$ & $14-34$ & $41.0(10.4)$ & -3.62 & 0.0003 \\
\hline & SDRG & 16 & 1 & $29.6(10.5)$ & $15-47$ & $64.0(14.5)$ & 13 & $19.0(6.2)$ & $12-34$ & $37.9(9.0)$ & -3.52 & 0.0004 \\
\hline \multirow{3}{*}{$\begin{array}{l}\text { Pairs with ATA } \\
\text { beyond a risk } \\
\text { period }^{\mathrm{c}}\end{array}$} & Total & 18 & 1 & $34.3(10.9)$ & $19-49$ & 72.2 (11.6) & 14 & $21.1(7.6)$ & $12-34$ & $47.2(5.2)$ & -3.66 & 0.0003 \\
\hline & FDRG & 10 & 1 & $38.3(9.3)$ & $23-49$ & $77.6(4.2)$ & 8 & $23.8(6.7)$ & $17-34$ & $48.6(5.7)$ & -2.65 & 0.0080 \\
\hline & SDRG & 8 & 0 & $29.4(11.2)$ & $19-47$ & $65.5(14.2)$ & 6 & $17.6(7.7)$ & $12-34$ & $45.4(4.2)$ & -2.52 & 0.0116 \\
\hline \multirow{3}{*}{$\begin{array}{l}\text { ATA controlled } \\
\text { pairs }\end{array}$} & Total & 26 & 3 & $30.7(11.0)$ & $15-49$ & $68.2(13.7)$ & 21 & $19.8(6.8)$ & $12-34$ & $41.6(10.1)$ & -4.33 & $<0.0001$ \\
\hline & FDRG & 14 & 3 & $34.0(11.1)$ & $15-49$ & 71.4 (11.6) & 11 & $21.4(6.9)$ & $14-34$ & $43.1(11.0)$ & -3.14 & 0.0017 \\
\hline & SDRG & 12 & 0 & $26.8(10.0)$ & $15-47$ & $64.3(15.4)$ & 10 & $18.0(6.5)$ & $12-34$ & $39.8(9.1)$ & -3.06 & 0.0022 \\
\hline
\end{tabular}

G1, Upper generation; G2, lower generation; MAO, Mean age at onset; MATA, Mean age at time of ascertainment; Z, Wilcoxon signed rank sum test (two-tailed); FDRG, first degree velatives group; SDRG, second degree relatives group

${ }^{\mathrm{a}} \mathrm{AO}$ of both members of pairs was $16-40$ years

${ }^{b}$ Both members of pairs were DSM-IV (Diagnostic and Statistical Manual of Mental Disorders, 4th ed.) schizophrenia patients

${ }^{\mathrm{c}}$ Subjects whose ATA was less than 40 years were excluded

${ }^{\mathrm{d}} \mathrm{ATA}$ in $\mathrm{G} 2$ was equal to or exceeded AO in G1

signed rank sum test and life table analyses for the analysis of statistical significance, and we also applied the Cox proportional hazard regression model to analyze the relationship between generation and AO, controlling for the cohort effect. To minimize ascertainment biases, pairwise comparison of AO in G1 and G2 was done in five ways: (1) All pairs. (2) Outliers excluded pairs, under the condition that the AO of both members of the pairs was 16-40 years. (3) Narrow diagnosis pairs, under the condition that both members of the pairs were DSM-IV schizophrenia patients. (4) Pairs with ATA beyond a risk period, under the condition that those subjects whose ATA was less than 40 years were excluded. (5) ATA controlled pairs, under the condition that the ATA in G2 was equal to or greater than the AO in G1.

To test the influence of transmission mode and sex difference, we compared the $\mathrm{AO}$ in $\mathrm{G} 1$ and $\mathrm{G} 2$ in regard to paternal and maternal transmission, and compared the AO in G1 and G2 in four schema: G1 females and G2 females; G1 females and G2 males; G1 males and G2 females; and G1 males and G2 males.

In all statistical analyses, we used the Statistical Package for the Social Sciences (SPSS) 6.1 program for the Macintosh (SPSS Japan Inc., Tokyo, Japan).

\section{Results}

Pairwise comparison

In pairwise comparisons, there were significant differences between mean $\mathrm{AO}$ in $\mathrm{G} 1$ and $\mathrm{G} 2$, with a significantly earlier mean $\mathrm{AO}$ in $\mathrm{G} 2$ in all of the pairs and in the two subgroups,
Table 4 Life table analysis of AO in schizophrenia

\begin{tabular}{llll}
\hline Generation & $\begin{array}{l}\text { Total number } \\
\text { of probards }\end{array}$ & FDRG & SDRG \\
\hline Mean AO in G1 & $33.4(n=38)$ & $37.4(n=24)$ & $27.9(n=15)$ \\
Mean AO in G2 & $20.8(n=39)$ & $21.0(n=27)$ & $20.4(n=12)$ \\
$\chi^{2}$ & 27.2 & 23.9 & 6.7 \\
df & 1 & 1 & 1 \\
$P^{b}$ (Wilcoxon test; & $<0.0001$ & $<0.0001$ & 0.0096 \\
$\quad$ two-tailed) & & & \\
\hline
\end{tabular}

AO, Age at onset of the disease (years); df, degrees of freedom. One patient in G1 was in both FDRG and SDRG

FDRG and SDRG. To exclude subjects with outlier AO of schizophrenia, we limited our sample to only those subjects in whom $\mathrm{AO}$ was $16-40$ years, and a significantly earlier AO in G2 was observed in all the pairs and in the two subgroups. Even when we limited our sample to a narrow diagnosis (DSM-IV schizophrenia), significantly earlier AO in $\mathrm{G} 2$ was observed in all the pairs and in the two subgroups (Table 3).

Life table analysis of AO

We performed a life table analysis with $\mathrm{AO}$ as the endpoint, for all the pairs, and for FDRG and SDRG. In all groups, subjects in $\mathrm{G} 2$ experienced significantly earlier AO than those in G1 (Table 4).

Cox proportional hazard regression model

We divided the subjects into two birth cohorts: those born after 1945 and cohorts born earlier, as, after the end of 
World War II in 1945, great cultural and socioeconomic changes took place in Japan. To estimate cohort effect, we used the Cox proportional hazard regression model, with birth cohort and generation membership as covariates, and with $\mathrm{AO}$ as the endpoint. The results showed a significant difference in $\mathrm{AO}$ between all subjects in $\mathrm{G} 1$ and all subjects in G2 $(\beta=0.966 ; P=0.008)$, even when we controlled for a significant birth cohort effect $(\beta=0.804 ; P=0.022)$.

Difference between paternal and maternal transmission

We compared the AO in two generations in paternal transmission (PT) and maternal transmission (MT) pairs (Table 5). Both MT and PT pairs showed significant differences in mean AO between G1 and G2 in all pairs, but, possibly because of the small number of pairs, PT pairs in FDRG did not show such differences.

Influence of sex difference on $\mathrm{AO}$

We compared the AO of G1 and G2 in the four previously described schema, and three showed a significant decrease in AO in G2 (Table 6).

\section{Discussion}

As has been pointed out, epidemiological research on anticipation involves various methodological issues, the foremost of which is that of bilineality, in which both paternal and maternal transmissions are present (Bassett and Honer 1994; Stöber et al. 1995; Thibaut et al. 1995; Gorwood et al. 1996). This issue involves the question of assortative mat- ing, and a major premise of anticipation research is the selection of pedigrees with unilineal transmissions of the disease. Stronger expression of the genes in bilineal G2 than unilineal G2 is expected. It is therefore necessary to rigorously exclude pedigrees with bilineal transmission. In the current study we selected only pairs for which both paternal and maternal family histories were complete, and excluded all pedigrees with possible bilineal transmission. Another effective method is to use operational criteria for family histories, such as the Family Interview for Genetic Studies (FIGS; National Institute of Mental Health, Bethesda, MDMolecular Genetics Initiative, 1991). We could not employ this method in the current study, but our data included substantial information, as previously mentioned.

To prove the presence of anticipation, the next problem is how to select a random sample, a task closely related to the problem of ascertainment biases. Therefore it is important that all subjects, especially those in G2, have passed through the period of risk for schizophrenia, because if they are selected at too early an age, it is possible that their siblings may eventually manifest the same disease as the proband after the investigation is completed.

As mentioned previously, Penrose (1948) reported that ascertainment biases could cause anticipation-like phenomena in several ways when the parent-offspring pair method is employed: (1) Affected parents with early AO are unlikely to be detected, because they are likely to have reduced fertility; (2) affected offspring with early AO and more severe symptoms are easily detected, because they are noticeable; (3) affected parent-offspring pairs with almost simultaneous onset are easily selected, because the information about each is easily available to researchers (Penrose 1948).

To minimize these biases, it is necessary to use pedigrees whose probands accurately represent the population of

Table 5 Paternal and maternal transmission of schizophrenia in AO

\begin{tabular}{|c|c|c|c|c|c|c|}
\hline & \multicolumn{3}{|c|}{ Paternal transmission pairs } & \multicolumn{3}{|c|}{ Maternal transmission pairs } \\
\hline & $\begin{array}{l}\text { Total } \\
(n=10)\end{array}$ & $\begin{array}{l}\text { FDRG } \\
(n=4)\end{array}$ & $\begin{array}{l}\text { SDRG } \\
(n=6)\end{array}$ & $\begin{array}{l}\text { Total } \\
(n=34)\end{array}$ & $\begin{array}{l}\text { FDRG } \\
(n=23)\end{array}$ & $\begin{array}{l}\text { SDRG } \\
(n=11)\end{array}$ \\
\hline $\mathrm{AO}$ in $\mathrm{G} 1(\mathrm{SD})$ & $36.6(10.7)$ & $37.5(10.0)$ & $36.0(12.1)$ & $34.7(11.3)$ & $38.3(10.7)$ & $27.0(8.3)$ \\
\hline $\mathrm{AO}$ in $\mathrm{G} 2(\mathrm{SD})$ & $22.6(5.6)$ & $23.8(5.6)$ & $21.8(6.1)$ & $19.6(5.9)$ & $20.6(5.9)$ & $17.5(5.6)$ \\
\hline $\mathrm{Z}$ & -2.80 & -1.83 & -2.21 & -5.01 & -4.14 & -2.93 \\
\hline$P$ value & 0.0050 & 0.0679 & 0.0273 & $<0.0001$ & $<0.0001$ & 0.0033 \\
\hline
\end{tabular}

AO, Age at onset of the disease, in years; $\mathrm{Z}$, Wilcoxon signed rank sum test (two-tailed)

Table 6 Pairwise comparisons of AO in G1 and G2 by sex

\begin{tabular}{lllll}
\hline & G1 Females-G2 females & G1 Females-G2 males & G1 Males-G2 females & G1 Males-G2 males \\
\hline Number of pairs & 15 & 17 & 3 & 9 \\
AO in G1 (SD) & $36.3(9.9)$ & $37.0(12.7)$ & $35.0(11.5)$ & $29.6(8.9)$ \\
AO in G2 (SD) & $20.9(5.4)$ & $19.8(6.5)$ & $22.7(5.5)$ & $19.1(6.3)$ \\
AO in G1 minus AO in G2 & $15.4(8.7)$ & $17.2(13.0)$ & $12.3(8.1)$ & $10.4(6.4)$ \\
Z & -3.41 & -3.48 & -1.60 & -2.67 \\
$P$ value & 0.0007 & 0.0005 & 0.1088 & 0.0077
\end{tabular}

AO, Age at onset of the disease (years); Z, Wilcoxon signed rank sum test (two-tailed) 
those with schizophrenia. In our sample, the mean age at the time of ascertainment in G1 was 64.8 years, and that in $\mathrm{G} 2$ was 38.3 years. This suggests that sample subjects in $\mathrm{G} 2$ had not completely passed through the risk age for the disease. Therefore, in our statistical analysis, we excluded those subjects whose ATA was 40 years or less. As shown, the $A O$ in $G 2$ was significantly lower than that in G1 in all the pairs, as well as in FDRG, and SDRG (Table 3). Our data seemed to be little affected by this type of ascertainment bias.

To consider subjects in G2, who are too young, another method is useful for estimating further morbidity risk. For example, Gottesman and Shields (1982) reported a $16.7 \%$ lifetime morbidity risk for the development of schizophrenia in siblings of a schizophrenic proband with one affected parent. In our sample, the risk for siblings of probands in G2 of FDRG was $11.9 \%$, less than the percentage reported by Gottesman and Shields. This indicates that about another $5 \%$ or less of the siblings may be affected later.

One ascertainment bias is caused by differences in ATA between G1 and G2, which leads to a greater chance of finding subjects with greater AO in G1. To control for this ascertainment bias, we calculated the "expected AO" proposed by Gorwood et al. (1996). The "expected AO" in G2 was estimated from the distribution of observed AO in G1 and the ATA in G2. As the number of our study subjects in G1 was small $(n=38)$, moving averages were used to estimate the probability and cumulative distribution of observed AO in G1 for the purpose of "smoothing". The mean "expected AO" in G2 was 26.87 years. Paired $t$-tests to compare the mean observed AO (20.85 years) and "expected AO" showed that in G2, the observed AO was significantly lower $(P<0.0001)$. This result was in accord with the finding of Gorwood et al. (1996).

Engström et al. (1995) proposed that to correct this ascertainment bias, parent-offspring pairs in which the AO of the parent was greater than the ATA of their offspring should be excluded. But this method has drawbacks. If the ATA in offspring is very young, for example, when offspring become probands at the time of their first episode, almost all pairs are excluded. When the ATA in offspring is very old, precise information often cannot be obtained. In our sample, the AO in G2 was significantly lower than that in G1, even when this method was employed. This finding shows that our sample was not greatly influenced by this ascertainment bias (Table 3).

Next, we have to consider the bias caused by the reduced fertility of parents with early onset (Penrose 1948). Fraser (1997) called this bias a "truncation bias" and distinguished it from ascertainment biases. In schizophrenia, this bias is more apparent than it is in mood disorders, because the band of AO in schizophrenia overlaps with marriageable age. The most effective method of reducing the influence of this bias is to examine not only parent-offspring pairs but also uncle- or aunt-nephew or -niece pairs. This bias is reduced considerably in comparisons between probands and their relatives in SDRG, especially if G1 subjects do not marry because of the disease. Moreover, by using such pairs, we can avoid environmental factors such as being raised by a parent with a mental disorder. We examined SDRG, and found a significant decrease of AO in G2 compared to that in G1.

It was difficult for us to completely exclude two other ascertainment biases suggested by Penrose (1948). first, subjects in G2 were detected as probands more readily than subjects in G1, and probands are likely to have severe symptoms and show an early AO. This may mimic anticipation. The best method for minimizing this bias is to perform pairwise comparison after exclusion of the pairs involving a proband of a pedigree; however, as our sample was small, we could not use this method. Second, the ascertainment bias related to pairs which show simultaneous onset is inevitably problematic for the retrospective study of anticipation. One method of avoiding this bias is to perform a long-term prospective study, but this type of study is difficult to carry out. However, recently, Bassett and Husted (1997) reanalyzed the data collected by Penrose during the period 1926-1944, and found that true anticipation was present in the transmission of schizophrenia, although the above ascertainment biases were observed.

Asherson et al. (1994) reported that their data, which showed a significant earlier AO in G2, was influenced by ascertainment bias. They found a significant correlation between parental AO and "anticipation", which they defined as the age difference between parental and offspring AO. Therefore, they maintained that the data represented regression to the mean, and not true anticipation. This view was supported by Yaw et al. (1996), but, in recent reports which used refined mathematical methods, other researchers have criticized its validity (McInnis et al. 1994; Hodge and Wickramaratne 1995). We performed a correlation analysis in the same way as did Asherson et al. (1994), and found a significant correlation between parental AO and "anticipation" (Spearman's correlation coefficient, $P<$ $0.001)$. However, in our data, only 1 of the 44 pairs showed negative anticipation. Moreover, in the pairs whose subjects in $\mathrm{G} 1$ showed early onset (AO, is younger than 23 years; $n$ $=6)$, the subjects in G2 had significantly lower AO than the subjects in G1 $(P=0.0277)$, and all these pairs showed positive anticipation. This result was different from those of Asherson et al. (1994) and Yaw et al. (1996) and suggested that our data did not show typical regression to the mean.

The cohort effect is reported as another important problem in the study of anticipation. Gershon et al. (1987) reported that in affective disorders, a significant difference in AO exists between cohorts born after 1940 and cohorts born earlier. Such a cohort effect may produce false anticipation. But, because these researchers estimated the cohort effect only in specific pedigrees, if anticipation were to exist in the pedigrees, its effects would be mixed with the cohort effect and would be likely to be judged the cohort effect. To estimate this bias, we performed an analysis using the Cox proportional hazard regression model. Although the results showed the presence of a cohort effect, there was a significantly earlier AO in G2 than in G1, even when we controlled for the cohort effect.

In our study, the AO in females was significantly higher than in males (female, 29.3 years; male, 24.1 years; 
Wilcoxon rank sum test, $P=0.0415)$. This finding is in keeping with that of previous reports. If the female-to-male ratio is large in G1 and small in G2, we may observe false anticipation. To test whether this female-to-male ratio could influence the results of this study, we compared the $\mathrm{AO}$ in $\mathrm{G} 1$ and $\mathrm{G} 2$ in four schema. In comparisons between G1 females and G2 females, G1 females and G2 males, and G1 males and G2 males, we observed a significant decrease in AO in G2 (Table 6). Because of the small sample size $(n=3)$, comparison between G1 males and G2 females did not show a significant result, but the mean difference between G1 and G2 in AO was more than 10 years, the same as in the other schema. Thus, we can assert that this bias did not significantly influence our sample.

Some researchers have mentioned that the presence of genetic imprinting is supporting evidence to prove anticipation, because in almost all unstable trinucleotide repeats diseases, genetic imprinting accompanies anticipation (Asherson et al. 1994; O'Donovan et al. 1995). However, in spinocerebellar ataxia type 2, a CAG repeats disease, anticipation is present, but molecular and clinical evidence for genetic imprinting has not been detected (Imbert et al. 1996; Petronis and Kennedy 1995). In our study, significant differences in mean AO were observed between G1 and G2 in all MT and PT pairs, although the differences were not significant in PT pairs in FDRG. However, we considered that this could result from too few PT pairs, and we observed no marked difference in AO between MT and PT pairs. For more conclusive observations regarding genetic imprinting, further investigation is needed.

Recently, a few researchers have presented negative views of anticipation in several diseases, including schizophrenia. Heiman et al. (1996) have reported that anticipation is caused by an ascertainment bias which is related to the differences in ATA in G1 and G2, based on the use of a computer simulation of pairs in FDRG. We performed not only a simple pairwise comparison but a pairwise comparison between ATA controlled pairs, and applied other methods such as life table analysis and comparison between observed and expected AO. Fraser (1997) has mentioned that between 1995 and 1996, anticipation was reported for 17 different conditions, and that all disorders with a variable AO occurring in successive generations and reducing fertility may show evidence of anticipation. But in our sample, there was a significant difference between mean $\mathrm{AO}$ in G1 and G2, even in SDRG. Fourteen of 15 G1 subjects in SDRG had no children, and even if we exclude one subject who has children, there was a significant difference $(P=0.0001)$. This result constitutes one of our objections to the supposed influence of a "truncation bias", which, according to Fraser, is the most influential bias for anticipation.

As mentioned above, several problems have been pointed out in the epidemiological study of anticipation. Although it is difficult to completely eliminate these problems, there are some valid ways to resolve each problem. We have demonstrated these solutions using statistical analysis, which showed an earlier AO in G2 than in G1. This result was observed for both FDRG and SDRG. We con- sider that these findings add new supportive evidence for epidemiological anticipation in schizophrenia.

The limitations of this study were that: (1) it was based on a chart review method, (2) our samples were small, and (3) in our sample, two ascertainment biases could not be ruled out. Therefore, in future studies we will aim to collect more subjects, and to conduct structured interviews.

A recent topic of interest in psychiatric genetics is the isolation of a new gene on chromosome 22q11.2-q13.1 encoding the potassium channel and containing polymorphic CAG repeats; this is thought to be a schizophrenia-related gene (Chandy et al. 1998). This suggests that one of the schizophrenia-related genes contains TRE, or that a subgroup of schizophrenia is associated with a gene with TRE. Although TRE is not always related to anticipation, it is necessary to cautiously continue the study of epidemiological anticipation in schizophrenia, and to relate it to the study of biological anticipation, including the molecular genetics of unstable DNA such as TRE.

Acknowledgements The authors thank Masaki Hayashida, Akira Hamada, Sumitaka Matsumoto, Kosuke Fujimaru, Takahiro Fukusako, Takahiro Tsujita, and Norio Niikawa for their help and advice.

YO was supported for the study by a Research Grant for Nervous and Mental Disorders from the Ministry of Health and Welfare, Japan, between 1993 and 1995 and 1996 and 1998. AI was supported for the study by a Grant from the Alumni Association of Nagasaki University School of Medicine in 1995.

\section{References}

Asherson P, Walsh C, Williams J, Sargeant M, Taylor C, Clements A, Gill M, Owen M, McGuffin P (1994) Imprinting and anticipation; Are they relevant to genetic studies of schizophrenia? Br J Psychiatry 164: 619-624

Ashley CT, Warren ST (1995) Trinucleotide repeat expansion and human disease. Annu Rev Genet 29: 703-728

Bassett AS, Honer WG (1994) Evidence for anticipation in schizophrenia. Am J Hum Genet 54: 864-870

Bassett AS, Husted J (1997) Anticipation or ascertainment bias in schizophrenia? Penrose's familial mental illness sample. Am J Hum Genet 60: 630-637

Broude NE, Chandra A, Smith CL (1997) Differential display of genome subsets containing specific interspersed repeats. Proc Natl Acad Sci U S A 94: 4548-4553

Chandy KG, Fantino E, Wittekindt O, Kalman K, Tong L-L, Ho T-H, Gutman GA, Croccq M-A, Gangli R, Nimgaonkar V, MorrisRosendahl DJ, Gargus JJ (1998) Isolation of a novel potassium channel gene hSKCa3 containing a polymorphic CAG repeat: a candidate for schizophrenia and bipolar disorder? Mol Psychiatry 3: 32-37

Dann JC, Laval SH, Loftus J, DeLisi LE, Smith A, Crow TJ (1996) Analysis of X-linked CAG repeats as candidate genes for schizophrenia. Psychiatr Gene 6: 153

Engström C, Thornlund A-S, Johansson E-L, Långström M, Chotai J, Adolfsson R, Nylander P-O (1995) Anticipation in unipolar affective disorder. J Affect Dis 35: 31-40

Fraser FC (1997) Trinucleotide repeats not the only cause of anticipation. Lancet 350: 459-460

Gershon ES, Hamovit JH, Guroff JJ, Nurnberger JI (1987) Birth-cohort change in manic and depressive disorders in relatives of bipolar and schizoaffective patients. Arch Gen Psychiatry 44: 314 319

Gorwood P, Leboyer M, Falissard B, Jay M, Rouillon F, Feingold J (1996) Anticipation in schizophrenia: new light on a controversial problem. Am J Psychiatry 153: 1173-1177 
Gottesman II, Shields J (1982) Schizophrenia: The epigenetic puzzle. Cambridge University Press, New York

Heiman GA, Hodge SE, Wickramaratne, Hsu H (1996) Age-atinterview bias in anticipation studies: computer simulations and an example with panic disorder. Psychiatr Genet 6: 61-66

Harper PS, Harley HG, Reardon W, Shaw DJ (1992) Anticipation in myotonic dystrophy, New light on an old problem. Am J Hum Genet 51: $10-16$

Hodge SE, Wickramaratne P (1995) Statistical pitfalls in detecting ageof-onset anticipation: the role of correlation in studying anticipation and detecting ascertainment bias. Psychiatr Genet 5: 43-47

Höweler CJ, Busch HFM, Geraedts JPM, Niermeijer MF, Staal A (1989) Anticipation in myotonic dystrophy: Fact or fiction? Brain 112: 779-797

Imbert G, Saudou F, Yvert G, Devys D, Trottier Y, Gaenier J-M, Weber C, Mandel J-L, Cancel G, Abbas N, Dürr A, Didierjean O, Stevanin G, Agid Y, Brice A (1996) Cloning of the gene for spinocerebellar ataxia 2 reveals a locus with high sensitivity to expanded CAG/glutamine repeats. Nature Genet 14: 285-291

Johnson JE, Cleary J, Ahsan H, Friedman JH, Malaspina D, Cloninger CR, Faraone SV, Tsuang MT, Kaufmann CA (1997) Anticipation in schizophrenia: biology or bias? Am J Med Genet 74: 275-280

Laurent C, Zander C, Thibaut F, Martinez M, Campion D, Jay M, Chavand O, Samolyk D, Cann H, Neri C, Mallet J (1996) Anticipation and $\mathrm{CAG} / \mathrm{CTG}$ repeat expansion detection in schizophrenia. Psychiatr Genet 6: 154

McInnis MG, McMahon FJ, Staine OC, Ross CA (1994) Reply to Petronis et al. (letter). Am J Hum Genet 55: 590-592

Nakamoto M, Takebayashi H, Kawaguchi Y, Narumiya S, Taniwaki M, Nakamura Y, Ishikawa Y, Akiguchi I, Kimura J, Kakizuka A (1997)
A CAG/CTG expansion in the normal population. Nature Genet 17: 385-386

O’Donovan MC, Guy C, Chaddock N, Murphy KC, Cardno AG, Jones LA, Owen MJ, McGuffin P (1995) Expanded CAG repeats in schzophrenia and bipolar disorder. Nature Genet 10: 380381

Petronis A, Bassett AS, Honer WG, Vincent JB, Tatuch Y, Sasaki T, Ying DJ, Klempan TA, Kennedy JL (1996) Search for unstable DNA in schizophrenia families with evidence for genetic anticipation. Am J Hum Genet 59: 905-911

Petronis A, Kennedy JL (1995) Unstable gene-unstable mind? Am J Psychiatry 152: 164-172

Penrose LS (1948) The problem of anticipation in pedigree of dystrophia myotonica. Ann Eugenics 14: 125-132

Ridley RM, Frith CD, Crow TJ, Conneally PM (1988) Anticipation in Huntington's disease is inherited through the male line but may originate in the female. J Med Genet 25: 589-595

Stöber G, Franzek E, Lesch KP, Beckmann H (1995) Periodic catatonia; a schizophrenic subtype with major gene effect and anticipation. Eur Arch Psychiatry Clin Neurosci 245: 135-141

Thibaut F, Martinez M, Petit M, Jay M, Campion D (1995) Further evidence for anticipation in schizophrenia. Psychiatry Res 59: 25-33

Vincent JB, Klempan T, Parikh SS, Sasaki T, Meltzer HY, Sirugo G, Cola P, Petronis A, Kennedy JL (1996) Frequency analysis of large CAG/CTG trinucleotide repeats in schizophrenia and bipolar affective disorder. Mol Psychiatry 1: 141-148

Yaw J, Myles-Worsley M, Hoff M, Holik J, Freedman R, Byerley W, Coon H (1996) Anticipation in multiplex schizophrenia pedigrees. Psychiatr Genet 6: 7-11 